\title{
Extracellular vesicles: structure, function, and potential clinical uses in renal diseases
}

\author{
F.T. Borges ${ }^{1,2}$, L.A. Reis ${ }^{1}$ and N. Schor ${ }^{1}$ \\ ${ }^{1}$ Divisão de Nefrologia, Departamento de Medicina, Universidade Federal Paulista, Escola Paulista de Medicina, \\ São Paulo, SP, Brasil \\ ${ }^{2}$ Programa de Pós-Graduação em Ciências da Saúde, Ciências Biológicas e da Saúde, Universidade Cruzeiro do Sul, São Paulo, SP, Brasil
}

\begin{abstract}
Interest in the role of extracellular vesicles in various diseases including cancer has been increasing. Extracellular vesicles include microvesicles, exosomes, apoptotic bodies, and argosomes, and are classified by size, content, synthesis, and function. Currently, the best characterized are exosomes and microvesicles. Exosomes are small vesicles (40-100 nm) involved in intercellular communication regardless of the distance between them. They are found in various biological fluids such as plasma, serum, and breast milk, and are formed from multivesicular bodies through the inward budding of the endosome membrane. Microvesicles are 100-1000 nm vesicles released from the cell by the outward budding of the plasma membrane. The therapeutic potential of extracellular vesicles is very broad, with applications including a route of drug delivery and as biomarkers for diagnosis. Extracellular vesicles extracted from stem cells may be used for treatment of many diseases including kidney diseases. This review highlights mechanisms of synthesis and function, and the potential uses of wellcharacterized extracellular vesicles, mainly exosomes, with a special focus on renal functions and diseases.
\end{abstract}

Key words: Extracellular vesicles; Cell biology; Kidney diseases; Diagnosis; Treatment

\section{Introduction}

The importance and role of extracellular vesicles, such as microvesicles and exosomes, are well documented in many diseases. Several studies have analyzed the effect of extracellular vesicles in cellular communication, but the different populations of extracellular vesicles are often presented in a confusing manner in the literature. Microvesicles are larger than exosomes (100-1000 nm) and are released into the extracellular space by outward budding of the cell membrane. On the other hand, exosomes are produced by a more complex inward budding of endosomes (1). Both microvesicles and exosomes are intercellular protein and RNA transporters and share other functions that are discussed in this review.

Other cellular vesicles include argosomes, blebs, and apoptotic bodies. Argosomes are exosome-like vesicles that contain morphogens, proteins that form a concentration gradient in the tissue, which is involved in signal transduction to convey cellular position during development in multicellular organisms. Argosomes differ from exosomes mainly in their function. They were found to be released from the basolateral membrane of wing disc cells of Drosophila melanogaster. The spread of their morphogens through epithelium promotes development $(2,3)$. It is suggested that they are involved in the direct transfer of materials between donor and recipient cells. Until recently, no standardized method of isolation or any specific marker for these extracellular vesicles was developed.

Apoptotic blebs are protrusive blisters formed when cellular plasma membrane delaminates from the cortical cytoskeleton, covering the entire surface of apoptotic cells. The formation of apoptotic blebs is a physical process that results from an increase in hydrostatic pressure following cellular contraction (4). This dynamic cyclic process of bleb formation and retraction can occur over sustained periods during the progression of programmed cell death. Apoptotic blebs become packed with cellular organelles and chromatin to form the basis of fragmentary membrane-clad apoptotic bodies. Some in

Correspondence: N. Schor, Divisão de Nefrologia, UNIFESP/EPM, Rua Botucatu, 740, 04023-900 São Paulo, SP, Brasil. Fax: +5511-5573-9652. E-mail: nestor@nefro.epm.br 
vitro studies have reported that inhibition of apoptotic blebbing significantly impaired corpse clearance by monocytes and macrophages $(5,6)$. Apoptotic bodies are the final consequences of cellular fragmentation. They are 1000-5000 $\mathrm{nm}$ extracellular vesicles that contain intact organelles, DNA, and histones (7). Apoptotic bodies do not come under the scope of this review, but more information about isolation and characteristics can be found in Table 1, which summarizes the differences between well-characterized extracellular vesicles, exosomes, microvesicles, and apoptotic bodies.

There is increasing interest in the study of the importance and function of extracellular vesicles and this review analyzes the mechanism of production and function, and potential clinical uses of extracellular vesicles in renal diseases.

\section{Exosomes: formation and release}

Exosomes are secreted by many cell types (8-10), and have been isolated from several physiological fluids such as sperm (11), urine (12), plasma (13), and bronchial lavage fluid (14). They have a diameter of $40-100 \mathrm{~nm}$, a homogeneous shape, with a density of $1.13-1.19 \mathrm{~g} / \mathrm{mL}$ in sucrose, and can be sedimented at 100,000 g. Most exosomes have an evolutionarily conserved set of proteins, including tetraspanins, Alix, and Tsg101, and have specific proteins that reflect their cellular source (1) (Table 1). They are formed from multivesicular bodies (MVBs), which are intracellular endosomal organelles, characterized by multiple intraluminal vesicles enclosed within a single outer membrane. MVBs are formed from early endosomes, which as prelysosomal structures belong to the degradative endosomal pathway of internalized proteins. They are now known to be involved in numerous endocytic and trafficking functions, including protein sorting, recycling, transport, storage, and release. Actually, some authors recognize two types of MVBs, one in the degradative pathway and another in the exocytosis or recycling pathway (15).

Early endosomes can interact with the Golgi apparatus and the endoplasmic reticulum. Exosomes can be formed by endocytosis of the early endosome membrane,

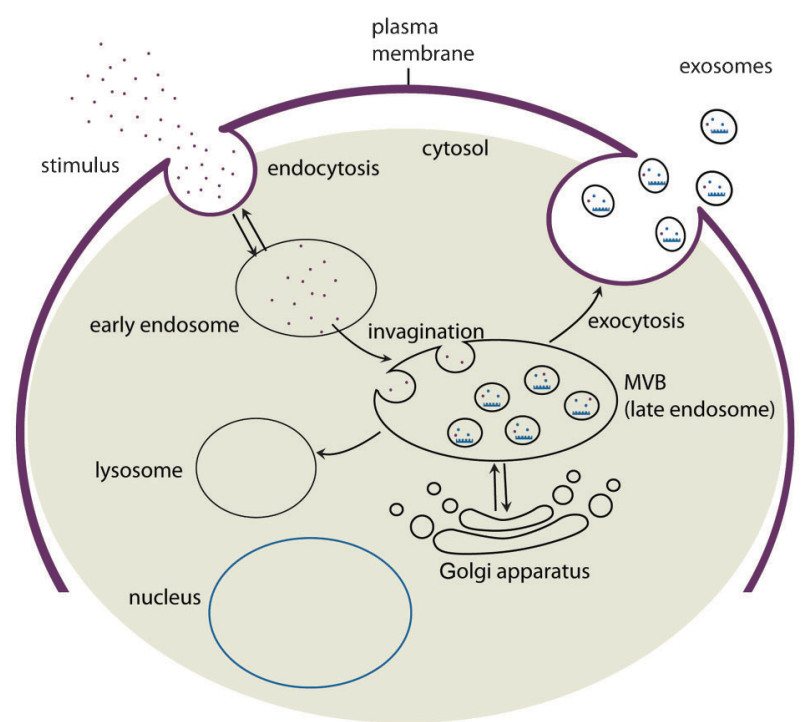

Figure 1. Early endosomes (EE) are formed by endocytosis following microenvironment modifications. The EE undergo maturation forming the late endosome or multivesicular body (MVB). During this process, EE communicate with the Golgi apparatus through bidirectional vesicle exchange. Inward budding of the membrane forms the intraluminal vesicles that will be released to the extracellular space as exosomes, or will fuse with lysosomes where an active degradation process will take place.

having a unique orientation of the involuted cytoplasmic side $(16,17)$ (Figure 1). Generation of MVBs as well as secretion of exosomes are mediated through the concerted action of endosomal complexes required for transport (ESCRT complexes). These protein complexes are involved in the recognition of ubiquitinated cargo by MVBs, as well as the invagination of the MVB outer membrane $(18,19)$.

The origin of exosomes suggests that their production is stimulated in response to alterations in the microenvironment. The formation of early endosomes and MVBs has been shown to increase upon signaling via growth factor receptors, suggesting that the cell adjusts exosome production according to its need $(12,20)$.

Table 1. Main differences between extracellular vesicles, in vitro isolation methods, cargo, size, and function.

\begin{tabular}{|c|c|c|c|}
\hline & Exosome & $\begin{array}{l}\text { Microvesicle, ectosome, } \\
\text { shedding vesicles }\end{array}$ & Apoptotic bodies \\
\hline Size & $40-100 \mathrm{~nm}$ & $100-1000 \mathrm{~nm}$ & $1000-5000 \mathrm{~nm}$ \\
\hline Markers & CD63, CD9, Alix, TSG 101, HSP 70 & $\begin{array}{l}\text { Anexin V, Flotillin-2, selectin, } \\
\text { integrin, CD40 metalloproteinase }\end{array}$ & Anexin V, DNA, histones \\
\hline Isolation method & $\begin{array}{l}\left.\text { Immunoprecipitation (ExoQuick }{ }^{\circledR}\right) \text {, } \\
\text { ultracentrifugation }(100,000-200,000 \mathrm{~g}) \text {, } \\
\text { ultracentrifugation with density gradient }\end{array}$ & $\begin{array}{l}\text { Ultracentrifugation } \\
(10,000-60,000 \mathrm{~g})\end{array}$ & No standardized protocol \\
\hline References & 49,53 & 54 & 55 \\
\hline
\end{tabular}




\section{Microvesicles: formation, release, and main characteristics}

Microvesicles are extracellular vesicles ranging in size from 100-1000 nm, with different shapes. Although some authors use the term microvesicles for extracellular vesicles in general, the main differences between exosomes and microvesicles depend on size, formation, and the secretion process. They are formed by regulated release of outward budding of the plasma membrane. Several authors use different markers for microvesicles like flotillin-2, selectins, integrins, metalloproteinases, and a high level of phosphatidylserine on the outer surface (Table 1). These extracellular vesicles can be isolated by ultracentrifugation (21), and it is not known if overlapping can occur between these two different populations of extracellular vesicles. There are some resemblances between them: both carry proteins, mRNA, and microRNA (miRNA) and are involved in cellular communication $(22,23)$, possibly through the horizontal transfer of genetic material, directly stimulating the target cell by transferring receptors or proteins.

\section{Extracellular vesicle functions}

\section{Cellular communication}

Exosomes could be a vehicle by which cells communicate with each other. This finding is supported by experiments where exosomes modulated the function of specific cell lines but not others (24). In addition, exosomes produced by one type of cell can stimulate another specific cell line. For example, exosomes from human bone marrow mesenchymal stem cells can stimulate proximal tubular cells to proliferate through the transfer of hepatocyte growth factor-1 receptor mRNA, suggesting the horizontal transfer of genetic material (25).

Exosomes mediate communication between neighboring cells through a paracrine mechanism and also through long-distance targeting via the systemic circulation similar to hormones (i.e., an endocrine mechanism). This observation is supported by the fact that exosomes have been obtained from various biological fluids such as urine, blood, and breast milk (26). In addition, exosomes extracted from mesenchymal stem cells in culture were administered systemically to animals and protected them against acute kidney injury (AKI), induced ischemia/ reperfusion (I/R) (27), and nephrotoxic antibiotics (28).

It was observed that about $60 \%$ of the proteins carried by these vesicles were obtained from exosomes of different cell lines (intestinal epithelial cells, T cells, B cells), suggesting that some proteins were constitutively present in these structures to mediate its functions (29). But also the presence of exosome proteins that were not present in the cytoplasm of the donor cell was observed, indicating that they were produced specifically for the mechanism of exosome-mediated communication or other functions (29)

Exosomes carry bioactive lipids like phospholipases $A 2, C$ and $D$, and prostaglandins that may be involved in the clearance of exosomes (30). Interestingly, no DNA has been found in exosomes, and the RNA they contain does not exactly reflect the quantity of miRNA and RNA in the cells where they originated.

Supporting the communication function, exosomes not only have the capacity to carry a large cargo load, but can protect the contents from degradative enzymes like RNase, trypsin, or chemical substances, due to its bi-lipid membrane $(31,32)$.

The recipient cell can take up this extracellular vesicle through different mechanisms. Exosomes carry membrane proteins that have binding affinity to ligands on the recipient cell membranes or the extracellular matrix, such as transferrin receptor, tumor necrosis factor receptors, lactadherins, integrins, and tetraspanin proteins (e.g., CD9, CD63, and CD81) (33). These membrane molecules may be involved in the homing of exosomes to a specific tissue or microenvironment (15).

Another mechanism of uptake involves fusion with recipient cell membrane, resulting in transfer of the contents of exosomes (mRNAs, miRNAs, proteins, and signaling molecules) or endocytosis (34-36). The mechanism by which these processes are regulated remains to be understood, but it is observed that exosome uptake correlated with intracellular and microenvironmental acidity (37).

Additionally, it was observed that the RNA carried by exosomes was taken up and translated in the recipient cell, demonstrating that they carry functional RNA (24). The findings support the hypothesis that exosomes can be secreted by a specific stimulus, transported to the recipient cell and taken up, and can stimulate the recipient cell.

Microvesicles formed by budding from the plasma membrane are also involved in intercellular communication. The main communication mechanism characterized in the kidney is mediated by microvesicles released from mesenchymal stem cells that reprogram injured kidney cells (38). But both of the extracellular vesicles, i.e., exosomes and microvesicles, share some characteristics such as the capability to reprogram the recipient cell.

\section{Extracellular vesicles and kidney diseases}

There are several functions suggested for the exosomes in kidney diseases. They may mediate the transfer of information during renal tubule hypertrophy after nephron loss. Enlargement of renal tubule cells, widening of the tubule lumen followed by an increase in singlenephron glomerular filtration rate, and the transport capacity for salt and water can be mediated by exosome signaling in downstream nephron segments (39). Proximal tubular cell proteins found in downstream segments of the nephron (collector ducts) were probably 
transported by exosomes, demonstrating the communication function. The protein or RNA content in exosomes can reflect the cellular origin of the extracellular vesicle, and alteration in the microenvironment indicates an early phase of injury (40), emphasizing the diagnostic potential of extracellular vesicles in kidney diseases.

A number of studies have reported the regenerative potential of microvesicles derived from mesenchymal or endothelial progenitor stem cells against acute kidney injury induced by glycerol (38), cysplatin (41), ischemia, and reperfusion (27) in animals. It was reported that exosomes from mesenchymal stem cells protected rats against nephrotoxic drugs like gentamicin (28).

Extracellular vesicles are involved in the pathophysiological development of diseases. We observed that exosomes from hypoxic proximal cells carrying tumor growth factor- $\beta$ mRNA could activate fibroblasts to proliferate and produce matrix proteins as another early mechanism of early fibroblast activation during fibrosis (20). Microvesicles also have an important function in the progression of diseases. The human renal carcinoma cells expressing CD105 release microvesicles that stimulate neoangiogenesis. This may be implicated in tumor progression, and in formation of a premetastatic niche in the lung (42). So extracellular vesicles are implicated in both the disease process and regeneration.

\section{Extracellular vesicles as potential biomolecules in diagnostic tools}

The potential of exosomes as diagnostic molecules is highly promising. Proteasome analysis of urinary exosomes has identified proteins from all segments of the nephron, including glomerular podocytes (podocin and podocalyxin), proximal tubules (megalin, cubilin, aquaporin-1, and type IV carbonic anhydrase), thick ascending limb of Henle (type $2 \mathrm{Na}-\mathrm{K}-2 \mathrm{Cl}$ cotransporter), distal convoluted tubule (thiazide-sensitive $\mathrm{Na}-\mathrm{Cl}$ cotransporter), and the collecting duct (aquaporin 2) (12). They have been shown to reflect acute kidney injury and are candidate diagnostic markers (43). In another study, the excretion of exosomes containing aquaporin-1 protein was decreased in rats subjected to I/R (up to $96 \mathrm{~h}$ ) but later returned to normal levels (after $480 \mathrm{~h}$ ). The same result was observed in patients after renal allograft transplantation, suggesting that aquaporin- 1 carried by exosomes in urine may allow early-to-late detection of renal cellular states after $\mathrm{I} / \mathrm{R}$-induced injury and the subsequent regeneration. So extracellular vesicles may be used to predict post-transplant AKI (delayed graft failure) (44).

In healthy humans, urinary exosome analysis has identified over 1000 proteins from different segments of the nephron, but, most importantly, 34 of them were implicated in many kidney diseases such as autosomal dominant polycystic kidney disease type 1 (polycystin-1), autosomal dominant and recessive nephrogenic diabetes (aquaporin-2), antenatal Bartter syndrome type 1 (Na-K$2 \mathrm{Cl}$ symport), and Gitelman's syndrome (thiazide-sensitive $\mathrm{Na}-\mathrm{Cl}$ cotransporter) (45). Nevertheless, additional studies are necessary to analyze these proteins in exosomes from patients with these diseases $(12,45,46)$.

It was observed that circulating miRNA is decreased in patients with chronic kidney disease and is correlated with the decrease in estimated glomerular filtration rate. The authors suggest that total and specific miRNA can be used as biomarkers of uremia (47).

As observed for exosomes, microvesicles have been investigated for potential use as diagnostic tools. In tumors, microvesicles could be used to determine the status of the tumor and mRNA could indicate the origin of the microvesicle (48).

\section{Exosomes as a drug delivery system}

More recently, the property of exosomes as vehicles for intercellular communication has been exploited for the delivery of therapeutic small interfering RNAs (siRNAs) to the brain and to provide alternative routes for drug delivery systems, much as a 'Trojan Horse' (49). Through this strategy, the HIV retrovirus exploits the machinery of exosome genesis for intercellular communication in the host cell to perform virus assembly and the cellular spread of infection in the host, independently of the binding of virus envelope protein to the cell receptor (50). Development of such a delivery system for therapeutic purposes faces many challenges, including ethical and technical issues; for example, choice of the best gene transfection method to introduce the RNA/protein of interest (cargo) into exosome-secreting cells (virus, lipofection, electroporation), how to target the cargo into the exosomes, the best technique to load exosomes with the drug of interest (electroporation or lipofection), and the cost of the process are a few of the concerns that need to be addressed.

On the contrary, exosomes have many unique attributes that make them an excellent choice as a drug delivery system: both protein and genetic material can be loaded into exosomes, they are well tolerated by the human body, have a long circulating half-life, are capable of membrane penetration, have intrinsic homing ability, and are amenable to membrane modifications (51). If we consider mesenchymal stem cell exosomes and microvesicles, the therapeutic potential improves. Since they are easily accessible in vitro, mesenchymal stem cells have a large capacity for expansion, are nonimmunogenic, and have the intrinsic therapeutic property of reducing tissue injury, as highlighted by different authors $(27,28,38,41,52)$.

Although research on the use of microvesicles/ exosomes for drug delivery in kidney diseases is still in its infancy, one study analyzed the potential of chemically modified $143 \mathrm{miR}$ transfected in THP-1 macrophages ex vivo, and microvesicles were isolated and injected into 
xenografted nude animals. The microvesicles were localized in serum, tumor, and kidney of the host animal. These experiments indicate that the cargo of microvesicles can be modified to deliver miRNA, and probably mRNA and proteins, to the kidney of the host (52).

Although this mechanism has an important therapeutic potential, more studies are still necessary to sufficiently address a number of issues related to safety and ethics.

\section{References}

1. Mathivanan S, Ji H, Simpson RJ. Exosomes: extracellular organelles important in intercellular communication. J Proteomics 2010; 73: 1907-1920, doi: 10.1016/j.jprot.2010. 06.006.

2. Greco V, Hannus M, Eaton S. Argosomes: a potential vehicle for the spread of morphogens through epithelia. Cell 2001; 106: 633-645, doi: 10.1016/S0092-8674(01)00484-6.

3. Robbins JR, Barth AI, Marquis H, de Hostos EL, Nelson WJ, Theriot JA. Listeria monocytogenes exploits normal host cell processes to spread from cell to cell. J Cell Biol 1999; 146: 1333-1350, doi: 10.1083/jcb.146.6.1333.

4. Wickman G, Julian L, Olson MF. How apoptotic cells aid in the removal of their own cold dead bodies. Cell Death Differ 2012; 19: 735-742, doi: 10.1038/cdd.2012.25.

5. Orlando KA, Stone NL, Pittman RN. Rho kinase regulates fragmentation and phagocytosis of apoptotic cells. Exp Cell Res 2006; 312: 5-15, doi: 10.1016/j.yexcr.2005.09.012.

6. Witasp E, Uthaisang W, Elenstrom-Magnusson C, Hanayama R, Tanaka M, Nagata S, et al. Bridge over troubled water: milk fat globule epidermal growth factor 8 promotes human monocyte-derived macrophage clearance of non-blebbing phosphatidylserine-positive target cells. Cell Death Differ 2007; 14: 1063-1065.

7. Kerr JF, Wyllie AH, Currie AR. Apoptosis: a basic biological phenomenon with wide-ranging implications in tissue kinetics. Br J Cancer 1972; 26: 239-257, doi: 10.1038/bjc. 1972.33.

8. Heijnen HF, Schiel AE, Fijnheer R, Geuze HJ, Sixma JJ. Activated platelets release two types of membrane vesicles: microvesicles by surface shedding and exosomes derived from exocytosis of multivesicular bodies and alpha-granules. Blood 1999; 94: 3791-3799.

9. Fevrier B, Vilette D, Archer F, Loew D, Faigle W, Vidal M, et al. Cells release prions in association with exosomes. Proc Natl Acad Sci U S A 2004; 101: 9683-9688, doi: 10. 1073/pnas.0308413101.

10. Wolfers J, Lozier A, Raposo G, Regnault A, Thery C, Masurier $\mathrm{C}$, et al. Tumor-derived exosomes are a source of shared tumor rejection antigens for CTL cross-priming. Nat Med 2001; 7: 297-303, doi: 10.1038/85438.

11. Sullivan R, Saez F, Girouard J, Frenette G. Role of exosomes in sperm maturation during the transit along the male reproductive tract. Blood Cells Mol Dis 2005; 35: 1-10, doi: 10.1016/j.bcmd.2005.03.005.

12. Pisitkun T, Shen RF, Knepper MA. Identification and proteomic profiling of exosomes in human urine. Proc Natl Acad Sci U S A 2004; 101: 13368-13373, doi: 10.1073/ pnas.0403453101.

\section{Conclusion and future directions}

The use of exosomes as diagnostic biomolecules in a wide range of diseases and as a therapeutic drug delivery system is highly promising. Nevertheless, more studies are required to understand the mechanism of exosome formation and release as well as their physiological and pathological functions in different organs and systems, including non-cancer-related kidney diseases.

13. Caby MP, Lankar D, Vincendeau-Scherrer C, Raposo G, Bonnerot C. Exosomal-like vesicles are present in human blood plasma. Int Immunol 2005; 17: 879-887, doi: 10.1093/ intimm/dxh267.

14. Admyre C, Grunewald J, Thyberg J, Gripenback S, Tornling G, Eklund A, et al. Exosomes with major histocompatibility complex class II and co-stimulatory molecules are present in human BAL fluid. Eur Respir J 2003; 22: 578-583, doi: 10.1183/09031936.03.00041703.

15. Mathivanan S, Simpson RJ. ExoCarta: A compendium of exosomal proteins and RNA. Proteomics 2009; 9: 49975000, doi: 10.1002/pmic.200900351.

16. Palay SL. The fine structure of secretory neurons in the preoptic nucleus of the goldish (Carassius auratus). Anat Rec 1960; 138: 417-443, doi: 10.1002/ar.1091380404.

17. Roizin L, Nishikawa K, Koizumi J, Keoseian S. The fine structure of the multivesicular body and their relationship to the ultracellular constituents of the central nervous system. J Neuropathol Exp Neurol 1967; 26: 223-249, doi: 10.1097| 00005072-196704000-00003.

18. Babst M, Katzmann DJ, Estepa-Sabal EJ, Meerloo T, Emr SD. Escrt-III: an endosome-associated heterooligomeric protein complex required for mvb sorting. Dev Cell 2002; 3: 271-282, doi: 10.1016/S1534-5807(02)00220-4.

19. Wollert T, Hurley JH. Molecular mechanism of multivesicular body biogenesis by ESCRT complexes. Nature 2010; 464: 864-869, doi: 10.1038/nature08849.

20. Borges FT, Melo SA, Ozdemir BC, Kato N, Revuelta I, Miller $\mathrm{CA}$, et al. TGF-beta1-containing exosomes from injured epithelial cells activate fibroblasts to initiate tissue regenerative responses and fibrosis. J Am Soc Nephrol 2013; 24: 385-392, doi: 10.1681/ASN.2012101031.

21. Gyorgy B, Modos K, Pallinger E, Paloczi K, Pasztoi M, Misjak $P$, et al. Detection and isolation of cell-derived microparticles are compromised by protein complexes resulting from shared biophysical parameters. Blood 2011; 117: e39-e48, doi: 10.1182/blood-2010-09-307595.

22. Souza-Schorey C, Clancy JW. Tumor-derived microvesicles: shedding light on novel microenvironment modulators and prospective cancer biomarkers. Genes Dev 2012; 26: 1287-1299, doi: 10.1101/gad.192351.112.

23. Skog J, Wurdinger T, van Rijn S, Meijer DH, Gainche L, Sena-Esteves M, et al. Glioblastoma microvesicles transport RNA and proteins that promote tumour growth and provide diagnostic biomarkers. Nat Cell Biol 2008; 10: 14701476, doi: 10.1038/ncb1800.

24. White IJ, Bailey LM, Aghakhani MR, Moss SE, Futter CE. EGF stimulates annexin 1-dependent inward vesiculation in 
a multivesicular endosome subpopulation. EMBO J 2006; 25: 1-12, doi: 10.1038/sj.emboj.7600759.

25. Tomasoni S, Longaretti L, Rota C, Morigi M, Conti S, Gotti $E$, et al. Transfer of growth factor receptor mRNA via exosomes unravels the regenerative effect of mesenchymal stem cells. Stem Cells Dev 2013; 22: 772-780, doi: 10.1089/ scd.2012.0266.

26. Lasser C, Alikhani VS, Ekstrom K, Eldh M, Paredes PT, Bossios A, et al. Human saliva, plasma and breast milk exosomes contain RNA: uptake by macrophages. J Trans/ Med 2011; 9: 9, doi: 10.1186/1479-5876-9-9.

27. Gatti S, Bruno S, Deregibus MC, Sordi A, Cantaluppi V, Tetta $\mathrm{C}$, et al. Microvesicles derived from human adult mesenchymal stem cells protect against ischaemia-reperfusion-induced acute and chronic kidney injury. Nephrol Dial Transplant 2011; 26: 1474-1483, doi: 10.1093/ndt/gfr015.

28. Reis LA, Borges FT, Simoes MJ, Borges AA, SinigagliaCoimbra R, Schor N. Bone marrow-derived mesenchymal stem cells repaired but did not prevent gentamicin-induced acute kidney injury through paracrine effects in rats. PLoS One 2012; 7: e44092, doi: 10.1371/journal.pone.0044092.

29. Valadi H, Ekstrom K, Bossios A, Sjostrand M, Lee JJ, Lotvall JO. Exosome-mediated transfer of mRNAs and microRNAs is a novel mechanism of genetic exchange between cells. Nat Cell Biol 2007; 9: 654-659, doi: 10.1038/ ncb1596.

30. Blanc L, Barres C, Bette-Bobillo P, Vidal M. Reticulocytesecreted exosomes bind natural IgM antibodies: involvement of a ROS-activatable endosomal phospholipase iPLA2. Blood 2007; 110: 3407-3416, doi: 10.1182/blood2007-04-085845.

31. Koga $\mathrm{Y}$, Yasunaga M, Moriya $\mathrm{Y}$, Akasu T, Fujita S, Yamamoto $S$, et al. Exosome can prevent RNase from degrading microRNA in feces. $J$ Gastrointest Oncol 2011; 2: 215-222.

32. Subra C, Grand D, Laulagnier K, Stella A, Lambeau G, Paillasse $M$, et al. Exosomes account for vesicle-mediated transcellular transport of activatable phospholipases and prostaglandins. J Lipid Res 2010; 51: 2105-2120, doi: 10.1194/Jr.M003657.

33. Chen TS, Lai RC, Lee MM, Choo AB, Lee CN, Lim SK. Mesenchymal stem cell secretes microparticles enriched in pre-microRNAs. Nucleic Acids Res 2010; 38: 215-224, doi: 10.1093/nar/gkp857.

34. Hemler ME. Tetraspanin proteins mediate cellular penetration, invasion, and fusion events and define a novel type of membrane microdomain. Annu Rev Cell Dev Biol 2003; 19: 397-422, doi: 10.1146/annurev.cellbio.19.111301.153609.

35. Tian T, Wang $Y$, Wang $H$, Zhu Z, Xiao Z. Visualizing of the cellular uptake and intracellular trafficking of exosomes by live-cell microscopy. J Cell Biochem 2010; 111: 488-496, doi: $10.1002 / j c b .22733$.

36. Feng D, Zhao WL, Ye YY, Bai XC, Liu RQ, Chang LF, et al. Cellular internalization of exosomes occurs through phagocytosis. Traffic 2010; 11: 675-687, doi: 10.1111/j.16000854.2010.01041.x.

37. Parolini I, Federici C, Raggi C, Lugini L, Palleschi S, De Milito $\mathrm{A}$, et al. Microenvironmental $\mathrm{pH}$ is a key factor for exosome traffic in tumor cells. J Biol Chem 2009; 284: 34211-34222, doi: 10.1074/jbc.M109.041152.

38. Bruno S, Grange C, Deregibus MC, Calogero RA, Saviozzi
$\mathrm{S}$, Collino $\mathrm{F}$, et al. Mesenchymal stem cell-derived microvesicles protect against acute tubular injury. J Am Soc Nephrol 2009; 20: 1053-1067, doi: 10.1681/ ASN.2008070798.

39. Fine LG, Schlondorff D, Trizna W, Gilbert RM, Bricker NS Functional profile of the isolated uremic nephron. Impaired water permeability and adenylate cyclase responsiveness of the cortical collecting tubule to vasopressin. J Clin Invest 1978; 61: 1519-1527, doi: 10.1172/JCl109072.

40. Brown D, Verbavatz JM, Valenti G, Lui B, Sabolic I. Localization of the CHIP28 water channel in reabsorptive segments of the rat male reproductive tract. Eur J Cell Biol 1993; 61: 264-273.

41. Bruno S, Grange C, Collino F, Deregibus MC, Cantaluppi V, Biancone L, et al. Microvesicles derived from mesenchymal stem cells enhance survival in a lethal model of acute kidney injury. PLoS One 2012; 7: e33115, doi: 10.1371/journal. pone.0033115

42. Grange C, Tapparo M, Collino F, Vitillo L, Damasco C Deregibus $\mathrm{MC}$, et al. Microvesicles released from human renal cancer stem cells stimulate angiogenesis and formation of lung premetastatic niche. Cancer Res 2011; 71: 5346-5356, doi: 10.1158/0008-5472.CAN-11-0241.

43. Zhou H, Pisitkun T, Aponte A, Yuen PS, Hoffert JD, Yasuda $\mathrm{H}$, et al. Exosomal Fetuin-A identified by proteomics: a novel urinary biomarker for detecting acute kidney injury. Kidney Int 2006; 70: 1847-1857, doi: 10.1038/sj.ki.5001874.

44. Sonoda H, Yokota-lkeda N, Oshikawa S, Kanno Y, Yoshinaga K, Uchida K, et al. Decreased abundance of urinary exosomal aquaporin-1 in renal ischemia-reperfusion injury. Am J Physiol Renal Physiol 2009; 297: F1006-F1016, doi: 10.1152/ajprenal.00200.2009.

45. Miranda KC, Bond DT, McKee M, Skog J, Paunescu TG, Da Slva $\mathrm{N}$, et al. Nucleic acids within urinary exosomes/ microvesicles are potential biomarkers for renal disease. Kidney Int 2010; 78: 191-199, doi: 10.1038/ki.2010.106.

46. Gonzales PA, Pisitkun T, Hoffert JD, Tchapyjnikov D, Star RA, Kleta R, et al. Large-scale proteomics and phosphoproteomics of urinary exosomes. J Am Soc Nephrol 2009; 20: 363-379, doi: 10.1681/ASN.2008040406.

47. Neal CS, Michael MZ, Pimlott LK, Yong TY, Li JY, Gleadle $\mathrm{JM}$. Circulating microRNA expression is reduced in chronic kidney disease. Nephrol Dial Transplant 2011; 26: 37943802, doi: $10.1093 / \mathrm{ndt} / \mathrm{gfr} 485$.

48. Tetta C, Bruno S, Fonsato V, Deregibus MC, Camussi G. The role of microvesicles in tissue repair. Organogenesis 2011; 7: 105-115, doi: 10.4161/org.7.2.15782.

49. Alvarez-Erviti L, Seow Y, Yin H, Betts C, Lakhal S, Wood MJ. Delivery of siRNA to the mouse brain by systemic injection of targeted exosomes. Nat Biotechnol 2011; 29: 341-345, doi: 10.1038/nbt.1807.

50. Gould SJ, Booth AM, Hildreth JE. The Trojan exosome hypothesis. Proc Natl Acad Sci U S A 2003; 100: 1059210597, doi: 10.1073/pnas.1831413100.

51. Lai RC, Yeo RW, Tan KH, Lim SK. Exosomes for drug delivery - a novel application for the mesenchymal stem cell. Biotechnol Adv 2013; 31: 543-551, doi: 10.1016/j.biote chadv.2012.08.008.

52. Akao $\mathrm{Y}$, lio $\mathrm{A}$, Itoh $\mathrm{T}$, Noguchi $\mathrm{S}$, Itoh $\mathrm{Y}$, Ohtsuki $\mathrm{Y}$, et al. Microvesicle-mediated RNA molecule delivery system using monocytes/macrophages. Mol Ther 2011; 19: 395-399, doi: 
10.1038/mt.2010.254.

53. Mathivanan S, Fahner CJ, Reid GE, Simpson RJ. ExoCarta 2012: database of exosomal proteins, RNA and lipids. Nucleic Acids Res 2012; 40: D1241-D1244, doi: 10.1093/ nar/gkr828.

54. Jayachandran M, Miller VM, Heit JA, Owen WG. Methodology for isolation, identification and characterization of microvesicles in peripheral blood. J Immunol Methods 2012; 375: 207-214, doi: 10.1016/j.jim.2011.10.012.

55. Hristov M, Erl W, Linder S, Weber PC. Apoptotic bodies from endothelial cells enhance the number and initiate the differentiation of human endothelial progenitor cells in vitro. Blood 2004; 104: 2761-2766, doi: 10.1182/blood-2003-103614. 